(n)

Eorberincisist Euler

Journal of Nonlinear Mathematical Physics

\title{
Integrable discrete autonomous quad-equations admitting, as generalized symmetries, known five-point differential-difference equations
}

Rustem N. Garifullin, Giorgio Gubbiotti, Ravil I. Yamilov

To cite this article: Rustem N. Garifullin, Giorgio Gubbiotti, Ravil I. Yamilov (2019) Integrable discrete autonomous quad-equations admitting, as generalized symmetries, known five-point differential-difference equations, Journal of Nonlinear Mathematical Physics 26:3, 333-357, DOI: https://doi.org/10.1080/14029251.2019.1613050

To link to this article: https://doi.org/10.1080/14029251.2019.1613050

Published online: 04 January 2021 


\title{
Integrable discrete autonomous quad-equations admitting, as generalized symmetries, known five-point differential-difference equations
}

\author{
Rustem N. Garifullin \\ Institute of Mathematics, Ufa Federal Research Centre, \\ Russian Academy of Sciences, \\ 112 Chernyshevsky Street, Ufa 450008, Russian Federation \\ rustem@matem.anrb.ru \\ Giorgio Gubbiotti* \\ School of Mathematics and Statistics, F07, The University of Sydney, \\ New South Wales 2006, Australia \\ giorgio.gubbiotti@sydney.edu.au \\ Ravil I. Yamilov \\ Institute of Mathematics, Ufa Federal Research Centre, \\ Russian Academy of Sciences, \\ 112 Chernyshevsky Street, Ufa 450008, Russian Federation \\ RvlYamilov@matem.anrb.ru
}

Received 26 October 2018

Accepted 1 February 2019

\begin{abstract}
In this paper we construct the autonomous quad-equations which admit as symmetries the five-point differential-difference equations belonging to known lists found by Garifullin, Yamilov and Levi. The obtained equations are classified up to autonomous point transformations and some simple non-autonomous transformations. We discuss our results in the framework of the known literature. There are among them a few new examples of both sine-Gordon and Liouville type equations.
\end{abstract}

Keywords: Integrability; Generalized symmetries; Quad-equations.

2000 Mathematics Subject Classification: 37L20, 37K10, 39A14

\section{Introduction}

In this paper we consider in discrete quad-equations. Quad-equations are four-point relations of the form:

$$
F\left(u_{n, m}, u_{n+1, m}, u_{n, m+1}, u_{n+1, m+1}\right)=0,
$$

for an unknown field $u_{n, m}$ depending on two discrete variables, i.e. $(n, m) \in \mathbb{Z}^{2}$. The function $F=$ $F(x, y, z, w)$ is assumed to be an irreducible multi-affine polynomial [6]. We recall that a polynomial is said to be multi-affine if it is affine with respect to all its variable, and it is said to be irreducible if it only admits trivial factors, i.e. complex constants. Moreover, by omitting the subscript $n, m$ we underline that it does not depend explicitly on the discrete variables, i.e. the equation (1.1) is assumed to be autonomous.

$\overline{{ }^{*} \text { Corresponding author }}$ 
It is known [20,29,31-33] that the generalized symmetries of quad-equations are given by differential-difference equations of the form:

$$
\begin{aligned}
\frac{\mathrm{d} u_{n, m}}{\mathrm{~d} t_{1}} & =\varphi_{n, m}\left(u_{n+k_{1}, m}, \ldots, u_{n-k_{1}^{\prime}, m}\right), \\
\frac{\mathrm{d} u_{n, m}}{\mathrm{~d} t_{2}} & =\psi_{n, m}\left(u_{n, m+k_{2}}, \ldots, u_{n, m-k_{2}^{\prime}}\right),
\end{aligned}
$$

where $k_{i}, k_{i}^{\prime} \in \mathbb{N}$. This means that one generalized symmetries depends only on shifts in the $n$ direction, while the other depends only on shifts in the $m$ direction. We note that usually differentialdifference equations are also addressed by the amount of points of the lattice that they are involving. In this sense the equations in (1.2) are a $k_{1}+k_{1}^{\prime}+1$-point differential-difference equation and a $k_{2}+k_{2}^{\prime}+1$-point differential-difference equation respectively.

The vast majority of quad-equations known in literature admits as generalized symmetries threepoint differential-difference equations in both directions [23, 29, 32, 36, 44, 45]. When the given quad-equation admits three-point generalized symmetries it can be interpreted as a Bäcklund transformation for these differential-difference equantions [28,29]. More recently, several examples with more complicated symmetry structure have been discovered [2,10,11, 14,33].

In the aforementioned papers the problem which was addressed was to find the generalized symmetries of a given quad-equation. If the generalized symmetries are given by integrable differentialdifference equations, then one can construct a whole hierarchy of generalized symmetries for the given quad-equation which is integrable according to the generalized symmetries method [46]. On the other hand, also the converse problem can be considered: fixed a differential-difference equation find the quad-equation admitting it as a generalized symmetry. This point of view was taken in [31], using one well-known five-point autonomous differential-difference equation. In [15] a similar analysis was carried out for a known class of integrable non-autonomous Volterra and Toda three-point differential-difference equations [30].

In this paper we are going to generalize the results of [15,31]: we will start from a known integrable autonomous five-point differential-differential equation and construct the quad-equation admitting it as generalized symmetry in the $n$ direction, i.e. of the form (1.2a). To this end we will use the classification of the five-point differential-difference equations given in $[18,19]$. The equations belonging to the classification in $[18,19]$ have the following form:

$$
\begin{aligned}
\frac{\mathrm{d} u_{k}}{\mathrm{~d} t} & =A\left(u_{k+1}, u_{k}, u_{k-1}\right) u_{k+2}+B\left(u_{k+1}, u_{k}, u_{k-1}\right) u_{k-2} \\
& +C\left(u_{k+1}, u_{k}, u_{k-1}\right),
\end{aligned}
$$

i.e. are linear in $u_{k \pm 2}$. We recall that in papers $[18,19]$ integrability is defined as the existence of an infinite hierarchy of generalized symmetries. These equations are divided in two classes, which we now describe explicitly. The first class, Class I was presented in [18] and contains all the equations of the form (1.3) such that the condition:

$$
A \neq \alpha\left(u_{k+1}, u_{k}\right) \alpha\left(u_{k}, u_{k-1}\right), \quad B \neq \beta\left(u_{k+1}, u_{k}\right) \beta\left(u_{k}, u_{k-1}\right),
$$

holds true for any functions $\alpha, \beta$. This Class contains seventeen equations. Equations belonging to this class are denoted by (E.x), where $\mathrm{x}$ is an Arabic number. The second class, Class II, was presented in [19] and contains all the equations of the form (1.3) such that the condition (1.4) does 
not hold, i.e. there exist functions $\alpha, \beta$ such that

$$
A=\alpha\left(u_{k+1}, u_{k}\right) \alpha\left(u_{k}, u_{k-1}\right), \quad B=\beta\left(u_{k+1}, u_{k}\right) \beta\left(u_{k}, u_{k-1}\right) .
$$

This class contains fourteen equations. Equations belonging to this class are denoted by $\left(\mathrm{E} . \mathrm{x}^{\prime}\right)$, where $\mathrm{x}$ is an Arabic number. For easier understanding of the results, we split the complete list into smaller Lists 1-6. In each List the equations are related to each other by autonomous non-invertible and non-point transformations or simple non-autonomous point transformations. Moreover, for sake of simplicity, since the equations are autonomous, in displaying the equations we will use the shorthand notation $u_{i}=u_{k+i}$.

List 1. Equations related to the double Volterra equation:

$$
\begin{aligned}
\frac{\mathrm{d} u_{0}}{\mathrm{~d} t} & =u_{0}\left(u_{2}-u_{-2}\right), \\
\frac{\mathrm{d} u_{0}}{\mathrm{~d} t} & =u_{0}^{2}\left(u_{2}-u_{-2}\right), \\
\frac{\mathrm{d} u_{0}}{\mathrm{~d} t} & =\left(u_{0}^{2}+u_{0}\right)\left(u_{2}-u_{-2}\right), \\
\frac{\mathrm{d} u_{0}}{\mathrm{~d} t} & =\left(u_{2}+u_{1}\right)\left(u_{0}+u_{-1}\right)-\left(u_{1}+u_{0}\right)\left(u_{-1}+u_{-2}\right), \\
\frac{\mathrm{d} u_{0}}{\mathrm{~d} t} & =\left(u_{2}-u_{1}+a\right)\left(u_{0}-u_{-1}+a\right) \\
& +\left(u_{1}-u_{0}+a\right)\left(u_{-1}-u_{-2}+a\right)+b, \\
\frac{\mathrm{d} u_{0}}{\mathrm{~d} t} & =u_{2} u_{1} u_{0}\left(u_{0} u_{-1}+1\right) \\
& -\left(u_{1} u_{0}+1\right) u_{0} u_{-1} u_{-2}+u_{0}^{2}\left(u_{-1}-u_{1}\right), \\
\frac{\mathrm{d} u_{0}}{\mathrm{~d} t} & =u_{0}\left[u_{1}\left(u_{2}-u_{0}\right)+u_{-1}\left(u_{0}-u_{-2}\right)\right], \\
\frac{\mathrm{d} u_{0}}{\mathrm{~d} t} & =u_{1} u_{0}^{2} u_{-1}\left(u_{2}-u_{-2}\right) .
\end{aligned}
$$

Transformations $\tilde{u}_{k}=u_{2 k}$ or $\tilde{u}_{k}=u_{2 k+1}$ turn equations (E.1)-(E.3) into the well-known Volterra equation and its modifications in their standard form. The other equations are related to the double Volterra equation(E.1) through some autonomous non-invertible nonpoint transformations. We note that equation (E.2') was presented in [4].

List 2. Linearizable equations:

$$
\begin{aligned}
\frac{\mathrm{d} u_{0}}{\mathrm{~d} t} & =(T-a)\left[\frac{\left(u_{1}+a u_{0}+b\right)\left(u_{-1}+a u_{-2}+b\right)}{u_{0}+a u_{-1}+b}+u_{0}+a u_{-1}+b\right] \\
& +c u_{0}+d, \\
\frac{\mathrm{d} u_{0}}{\mathrm{~d} t} & =\frac{u_{2} u_{0}}{u_{1}}+u_{1}-a^{2}\left(u_{-1}+\frac{u_{0} u_{-2}}{u_{-1}}\right)+c u_{0} .
\end{aligned}
$$

In both equations $a \neq 0$, in (E.7) $(a+1) d=b c$, and $T$ is the translation operator $T f_{k}=f_{k+1}$. 
R.N. Garifullin et al. / Integrable quad-equations admitting known five-point generalized symmetries

Both equations of List 2 are related to the linear equation:

$$
\frac{\mathrm{d} u_{0}}{\mathrm{~d} t}=u_{2}-a^{2} u_{-2}+\frac{c}{2} u_{0}
$$

through an autonomous non-invertible non-point transformations. We note that (E.7) is linked to (1.6) with a transformation which is implicit in both directions, see [18] for more details.

List 3. Equations related to a generalized symmetry of the Volterra equation:

$$
\begin{aligned}
\frac{\mathrm{d} u_{0}}{\mathrm{~d} t} & =u_{0}\left[u_{1}\left(u_{2}+u_{1}+u_{0}\right)-u_{-1}\left(u_{0}+u_{-1}+u_{-2}\right)\right] \\
& +c u_{0}\left(u_{1}-u_{-1}\right), \\
\frac{\mathrm{d} u_{0}}{\mathrm{~d} t} & =\left(u_{0}^{2}-a^{2}\right)\left[\left(u_{1}^{2}-a^{2}\right)\left(u_{2}+u_{0}\right)-\left(u_{-1}^{2}-a^{2}\right)\left(u_{0}+u_{-2}\right)\right] \\
& +c\left(u_{0}^{2}-a^{2}\right)\left(u_{1}-u_{-1}\right), \\
\frac{\mathrm{d} u_{0}}{\mathrm{~d} t} & =\left(u_{1}-u_{0}+a\right)\left(u_{0}-u_{-1}+a\right)\left(u_{2}-u_{-2}+4 a+c\right)+b, \\
\frac{\mathrm{d} u_{0}}{\mathrm{~d} t} & =u_{0}\left[u_{1}\left(u_{2}-u_{1}+u_{0}\right)-u_{-1}\left(u_{0}-u_{-1}+u_{-2}\right)\right], \\
\frac{\mathrm{d} u_{0}}{\mathrm{~d} t} & =\left(u_{0}^{2}-a^{2}\right)\left[\left(u_{1}^{2}-a^{2}\right)\left(u_{2}-u_{0}\right)+\left(u_{-1}^{2}-a^{2}\right)\left(u_{0}-u_{-2}\right)\right], \\
\frac{\mathrm{d} u_{0}}{\mathrm{~d} t} & =\left(u_{1}+u_{0}\right)\left(u_{0}+u_{-1}\right)\left(u_{2}-u_{-2}\right) .
\end{aligned}
$$

These equations are related between themselves by some transformations, for more details see [19]. Moreover equations (E.3',E.4',E.5') are the generalized symmetries of some known three-point autonomous differential-difference equations [46].

List 4. Equations of the relativistic Toda type:

$$
\begin{aligned}
\frac{\mathrm{d} u_{0}}{\mathrm{~d} t} & =\left(u_{0}-1\right)\left(\frac{u_{2}\left(u_{1}-1\right) u_{0}}{u_{1}}-\frac{u_{0}\left(u_{-1}-1\right) u_{-2}}{u_{-1}}-u_{1}+u_{-1}\right) \\
\frac{\mathrm{d} u_{0}}{\mathrm{~d} t} & =\frac{u_{2} u_{1}^{2} u_{0}^{2}\left(u_{0} u_{-1}+1\right)}{u_{1} u_{0}+1}-\frac{\left(u_{1} u_{0}+1\right) u_{0}^{2} u_{-1}^{2} u_{-2}}{u_{0} u_{-1}+1} \\
& -\frac{\left(u_{1}-u_{-1}\right)\left(2 u_{1} u_{0} u_{-1}+u_{1}+u_{-1}\right) u_{0}^{3}}{\left(u_{1} u_{0}+1\right)\left(u_{0} u_{-1}+1\right)} \\
\frac{\mathrm{d} u_{0}}{\mathrm{~d} t} & =\left(u_{1} u_{0}-1\right)\left(u_{0} u_{-1}-1\right)\left(u_{2}-u_{-2}\right) .
\end{aligned}
$$

Equation $\left(\mathrm{E} .13^{\prime}\right)$ was known $[12,14]$ to be is a relativistic Toda type equation. Since in [18] it was shown that the equations of List 4 are related through autonomous non-invertible non-point transformations, it was suggested that (E.9) and (E.10) should be of the same type. Finally, we note that equation (E.9) appeared in [1] earlier than in [18].

List 5. Equations related to the Itoh-Narita-Bogoyavlensky (INB) equation:

$$
\frac{\mathrm{d} u_{0}}{\mathrm{~d} t}=u_{0}\left(u_{2}+u_{1}-u_{-1}-u_{-2}\right)
$$


R.N. Garifullin et al. / Integrable quad-equations admitting known five-point generalized symmetries

$$
\begin{aligned}
\frac{\mathrm{d} u_{0}}{\mathrm{~d} t} & =\left(u_{2}-u_{1}+a\right)\left(u_{0}-u_{-1}+a\right) \\
& +\left(u_{1}-u_{0}+a\right)\left(u_{-1}-u_{-2}+a\right) \\
& +\left(u_{1}-u_{0}+a\right)\left(u_{0}-u_{-1}+a\right)+b, \\
\frac{\mathrm{d} u_{0}}{\mathrm{~d} t} & =\left(u_{0}^{2}+a u_{0}\right)\left(u_{2} u_{1}-u_{-1} u_{-2}\right), \\
\frac{\mathrm{d} u_{0}}{\mathrm{~d} t} & =\left(u_{1}-u_{0}\right)\left(u_{0}-u_{-1}\right)\left(\frac{u_{2}}{u_{1}}-\frac{u_{-2}}{u_{-1}}\right), \\
\frac{\mathrm{d} u_{0}}{\mathrm{~d} t} & =u_{0}\left(u_{2} u_{1}-u_{-1} u_{-2}\right), \\
\frac{\mathrm{d} u_{0}}{\mathrm{~d} t} & =\left(u_{1}-u_{0}+a\right)\left(u_{0}-u_{-1}+a\right)\left(u_{2}-u_{1}+u_{-1}-u_{-2}+2 a\right)+b, \\
\frac{\mathrm{d} u_{0}}{\mathrm{~d} t} & =u_{0}\left(u_{1} u_{0}-a\right)\left(u_{0} u_{-1}-a\right)\left(u_{2} u_{1}-u_{-1} u_{-2}\right), \\
\frac{\mathrm{d} u_{0}}{\mathrm{~d} t} & =\left(u_{1}+u_{0}\right)\left(u_{0}+u_{-1}\right)\left(u_{2}+u_{1}-u_{-1}-u_{-2}\right) .
\end{aligned}
$$

Equation (E.11) is the well-known INB equation [8,27,34]. Equations (E.12) with $a=0$ and (E.13) with $a=0$ are simple modifications of the INB and were presented in [33] and [9], respectively. Equation (E.13) with $a=1$ has been found in [40]. Up to an obvious linear transformation, it is equation (17.6.24) with $m=2$ in [40]. Equation (E. $\left.9^{\prime}\right)$ is a well-known modification of INB equation (E.11), found by Bogoyalavlesky himself [8]. Finally, equation (E.11') with $a=0$ was considered in [4]. All the equations in this list can be reduced to the INB equation using autonomous non-invertible non-point transformations. Moreover, equations (E.12),(E.14) and (E.9') are related through non-invertible transformations to the equation:

$$
\frac{\mathrm{d} u_{0}}{\mathrm{~d} t}=\left(u_{2}-u_{0}\right)\left(u_{1}-u_{-1}\right)\left(u_{0}-u_{-2}\right)
$$

We will also study below equation (1.7), see Remark 2.2 in Section 2.

List 6. Other equations:

$$
\begin{aligned}
\frac{\mathrm{d} u_{0}}{\mathrm{~d} t} & =u_{0}^{2}\left(u_{2} u_{1}-u_{-1} u_{-2}\right)-u_{0}\left(u_{1}-u_{-1}\right), \\
\frac{\mathrm{d} u_{0}}{\mathrm{~d} t} & =\left(u_{0}+1\right)\left[\frac{u_{2} u_{0}\left(u_{1}+1\right)^{2}}{u_{1}}-\frac{u_{-2} u_{0}\left(u_{-1}+1\right)^{2}}{u_{-1}}+\left(1+2 u_{0}\right)\left(u_{1}-u_{-1}\right)\right], \\
\frac{\mathrm{d} u_{0}}{\mathrm{~d} t} & =\left(u_{0}^{2}+1\right)\left(u_{2} \sqrt{u_{1}^{2}+1}-u_{-2} \sqrt{u_{-1}^{2}+1}\right), \\
\frac{\mathrm{d} u_{0}}{\mathrm{~d} t} & =u_{1} u_{0}^{3} u_{-1}\left(u_{2} u_{1}-u_{-1} u_{-2}\right)-u_{0}^{2}\left(u_{1}-u_{-1}\right) .
\end{aligned}
$$

Equation (E.15) has been found in [42] and it is called the discrete Sawada-Kotera equation $[2,42]$. Equation (E.14') is a simple modification of the discrete Sawada-Kotera equation (E.15). Equation (E.16) has been found in [1] and is related to (E.15). On the other hand equation (E.17) has been found as a result of the classification in [18] and seems to be a new equation. It was shown in [16] that equation (E.17) is a discrete analogue of the Kaup-Kupershmidt equation. Then we will refer to equation (E.17) as the discrete 
Kaup-Kupershmidt equation. No transformation into known equations of equation (E.17) is known.

As a result we will obtain several examples of autonomous nontrivial quad-equations. By nontrivial equations we mean non-degenerate, irreducible and nonlinear equations. Moreover we consider as trivial also the equations

$$
\begin{aligned}
& u_{n+1, m+1} u_{n, m+1}=\kappa_{1} u_{n+1, m} u_{n, m}, \\
& u_{n+1, m+1} u_{n, m}=\kappa_{2} u_{n+1, m} u_{n, m+1},
\end{aligned}
$$

which are equavalent to a linear one up to the transcendental transformation $u_{n, m}=\exp v_{n, m}$. The resulting equations belong to two different types of equations: they can be either Liouville type LT or sine-Gordon type sGT. Liouville type equations are quad-equation which are Darboux integrable, i.e. they possess first integrals, one containing only shifts in the first direction and the other containing only shifts in the second direction. This means that there exist two functions:

$$
\begin{aligned}
& W_{1}=W_{1, n, m}\left(u_{n+l_{1}, m}, u_{n+l_{1}+1, m}, \ldots, u_{n+k_{1}, m}\right), \\
& W_{2}=W_{2, n, m}\left(u_{n, m+l_{2}}, u_{n, m+l_{2}+1}, \ldots, u_{n, m+k_{2}}\right),
\end{aligned}
$$

where $l_{1}<k_{1}$ and $l_{2}<k_{2}$ are integers, such that the relations

$$
\begin{aligned}
& \left(T_{n}-\mathrm{Id}\right) W_{2}=0, \\
& \left(T_{m}-\mathrm{Id}\right) W_{1}=0
\end{aligned}
$$

hold true identically on the solutions of (1.1). By $T_{n}, T_{m}$ we denote the shift operators in the first and second directions, i.e. $T_{n} h_{n, m}=h_{n+1, m}, T_{m} h_{n, m}=h_{n, m+1}$, and by Id we denote the identity operator $\operatorname{Id} h_{n, m}=h_{n, m}$. The number $k_{i}-l_{i}$, where $i=1,2$, is called the order of the first integral $W_{i}$. On the other hand a quad-equation is said to be of sine-Gordon type if it is not Darboux integrable and possess two generalized symmetries of the form (1.2). Such equations are usually integrable by the inverse scatteting method.

We underline that, by construction, all the equations we are going to produce will possess a generalized symmetry in the $n$ direction (1.2a) which is not sufficient for integrability. To prove that the equation, we are going to produce, are LT or sGT we will either identify them with known equations, or present their first integrals in the sense of (1.9) or a generalized symmetry in the $m$ direction (1.2b).

The plan of the paper is then the following: In Section 2 we discuss the theoretical background which allows us to make the relevant computations. In Section 3 we enumerate all the quad-equations of the form (1.1) which corresponds to the equations of the Class I and II and describe their properties. Finally in Section 4 we give a summary of the work and some outlook for future works in the field. 


\section{The method}

The most general autonomous multi-affine quad-equation has the following form:

$$
\begin{gathered}
F \equiv u_{n+1, m+1} u_{n, m+1}\left(a_{1} u_{n, m} u_{n+1, m}+a_{2} u_{n, m}+a_{3} u_{n+1, m}+a_{4}\right) \\
+u_{n+1, m+1}\left(b_{1} u_{n, m} u_{n+1, m}+b_{2} u_{n, m}+b_{3} u_{n+1, m}+b_{4}\right) \\
+u_{n, m+1}\left(c_{1} u_{n, m} u_{n+1, m}+c_{2} u_{n, m}+c_{3} u_{n+1, m}+c_{4}\right) \\
+d_{1} u_{n, m} u_{n+1, m}+d_{2} u_{n, m}+d_{3} u_{n+1, m}+d_{4}=0 .
\end{gathered}
$$

From (1.2a) and (1.3) we have that the most general symmetry in the $n$ direction of the form (1.2a) belonging to Class I and II can be written as:

$$
\begin{aligned}
\dot{u}_{n, m} & =a\left(u_{n+1, m}, u_{n, m}, u_{n-1, m}\right) u_{n+2, m}+b\left(u_{n+1, m}, u_{n, m}, u_{n-1, m}\right) u_{n-2, m} \\
& +c\left(u_{n+1, m}, u_{n, m}, u_{n-1, m}\right) .
\end{aligned}
$$

We have then the following general result, analogous to Theorem 2 in [15]:

Theorem 2.1. If the autonomous quad-equation (2.1) admits a generalized symmetry of the form (2.2) then it has the following form:

$$
\begin{aligned}
\widehat{F} & \equiv \alpha u_{n+1, m+1} u_{n, m+1}+u_{n+1, m+1}\left(\beta_{1} u_{n, m}+\beta_{2}\right)+u_{n, m+1}\left(\gamma_{1} u_{n+1, m}+\gamma_{2}\right) \\
& +\delta_{1} u_{n, m} u_{n+1, m}+\delta_{2} u_{n, m}+\delta_{3} u_{n+1, m}+\delta_{4}=0 .
\end{aligned}
$$

Proof. By multi-linearity we can always solve (2.1) with respect to $u_{n+1, m+1}$ and write:

$$
u_{n+1, m+1}=f\left(u_{n+1, m}, u_{n, m}, u_{n, m+1}\right) .
$$

From Theorem 2 in [31] we have that a quad-equation admits a generalized symmetry of the form (1.2a) with $k_{1}=-k_{1}^{\prime}=2$ if the following conditions are satisfied:

$$
\begin{aligned}
\left(T_{n}-T_{n}^{-1}\right) \log \frac{\partial f}{\partial u_{n+1, m}} & =\left(1-T_{m}\right) \log \frac{\partial \varphi}{\partial u_{n+2, m}}, \\
\left(T_{n}^{-2}-1\right) \log \left(\frac{\partial f}{\partial u_{n, m}} / \frac{\partial f}{\partial u_{n, m+1}}\right) & =\left(1-T_{m}\right) \log \frac{\partial \varphi}{\partial u_{n-2, m}} .
\end{aligned}
$$

In (2.5) we suppressed the indices $n, m$ in $\varphi$ since we are dealing with autonomous differentialdifference equations. Now from (2.2) we have:

$$
\frac{\partial \varphi}{\partial u_{n+2, m}}=a\left(u_{n+1, m}, u_{n, m}, u_{n-1, m}\right), \quad \frac{\partial \varphi}{\partial u_{n-2, m}}=b\left(u_{n+1, m}, u_{n, m}, u_{n-1, m}\right) .
$$

From equation (2.6) we have differentiating (2.5a) w.r.t. $u_{n+2, m}$ :

$$
0=\frac{\partial}{\partial u_{n+2, m}} T_{n} \log \frac{\partial f}{\partial u_{n+1, m}}=-2 T_{n}\left(\frac{\partial^{2} F / \partial u_{n+1, m+1} \partial u_{n+1, m}}{\partial F / \partial u_{n+1, m+1}}\right),
$$

where we used the implicit function theorem and the fact that $F$ is multi-affine (2.1). In the same way differentiating (2.5b) w.r.t $u_{n-2, m}$ we get:

$$
0=\frac{\partial}{\partial u_{n-2, m}} T_{n}^{-2} \log \left(\frac{\partial f}{\partial u_{n, m}} / \frac{\partial f}{\partial u_{n, m+1}}\right)=-2 T_{n}^{-2}\left(\frac{\partial^{2} F / \partial u_{n, m+1} \partial u_{n, m}}{\partial F / \partial u_{n, m+1}}\right) .
$$


Therefore we get the two conditions:

$$
\frac{\partial^{2} F}{\partial u_{n+1, m+1} \partial u_{n+1, m}}=\frac{\partial^{2} F}{\partial u_{n, m+1} \partial u_{n, m}}=0 .
$$

Working out explicitly the conditions in (2.9) and using (2.1) we obtain:

$$
a_{1}=a_{2}=a_{3}=b_{1}=b_{3}=c_{1}=c_{2}=0 .
$$

Relabeling the parameters as

$$
a_{4} \rightarrow \alpha, \quad b_{2} \rightarrow \beta_{1}, \quad b_{4} \rightarrow \beta_{2}, \quad c_{3} \rightarrow \gamma_{1}, \quad c_{4} \rightarrow \gamma_{2}, \quad d_{i} \rightarrow \delta_{i},
$$

equation (2.3) follows.

Remark 2.1. We note that the condition (2.9) is the same as formula (29) in [15] even though the class of considered differential-difference equation is different.

Remark 2.2. It can be proved in a similar way that Theorem 2.1 is valid for generalized symmetries of the form (1.2a) satisfying the conditions $k_{1}^{\prime}=k_{1}$ and

$$
\frac{\partial^{2} \varphi_{n, m}}{\partial u_{n+k_{1}}^{2}}=0, \quad \frac{\partial^{2} \varphi_{n, m}}{\partial u_{n-k_{1}}^{2}}=0 .
$$

Theorem 2.1 tells us that the most general form of a quad-equation admitting a five-point generalized symmetry in the $n$ direction of the form (2.2) is given by (2.3). At this point we can pick up any of the members of Class I and II and follow the scheme of [18, App. B], i.e. we fix a specific form of $a, b$ and $c$ in (2.2). We beging by imposing the exponential integrability conditions (2.5a) and $(2.5 \mathrm{~b})$ and finally we impose the symmetry condition:

$$
\sum_{i, j \in\{0,1\}} \varphi\left(u_{n+2+i, m+j}, \ldots, u_{n-2+i, m+j}\right) \frac{\partial \widehat{F}}{\partial u_{n+i, m+j}}=0
$$

which must be satisfied on all the solutions of (2.3). Then we express in (2.13) the functions $u_{n+2, m+1}, u_{n+1, m+1}$ and $u_{n-1, m+1}$ in terms of the independent variables

$$
u_{n+2, m}, u_{n+1, m}, u_{n, m}, u_{n-1, m}, u_{n, m+1} .
$$

Taking the numerator of (2.13) we obtain a polynomial in the independent variable (2.14). This polynomial must be identically zero, so we can equate to zero all its coefficients.

This lead us to a system of algebraic equation in the coefficients of the multi-affine function (2.3). Doing so we reduce the problem of finding a quad-equation admitting a given five-point symmetry of the form (2.2) to the problem of solving system of algebraic equations. Such system can be solved using a Computer Algebra System like Maple, Mathematica or Reduce. Amongst the possible solutions of the system we choose the non-degenerate ones. The non-degeneracy condition is the following one:

$$
\frac{\partial F}{\partial u_{n+1, m+1}} \frac{\partial f}{\partial u_{n+1, m}} \frac{\partial f}{\partial u_{n, m+1}} \frac{\partial f}{\partial u_{n, m}} \neq 0 .
$$

Note that this non-degeneracy condition includes the requirement that a quad-equation must be given by an irreducible multi-affine polynomial, see the introduction. 
Remark 2.3. Several equations in Class I and II, e.g. (E.5) or (E.5'), depend on some parameters. Depending on the value of the parameters there can be, in principle, different quad-equations admitting the given differential-difference equation as five-point generalized symmetries. When possible, in order to avoid ambiguities and simplify the problem, we use some simple autonomous transformations to fix the values of some parameters. The remaining free parameters are then treated as unknown coefficients in the system of algebraic equations. We will describe these subcases when needed in the next section.

The number of the resulting equations is then reduced using some point transformations. These point transformations are essentially of two different kind: non-autonomous transformations of the dependent variable $u_{n, m}$ :

$$
\begin{gathered}
u_{n, m} \rightarrow(-1)^{m} u_{n, m}, \\
u_{n, m} \rightarrow\left(\frac{1+\mathrm{i} \sqrt{3}}{2}\right)^{m} u_{n, m}, \\
u_{n, m} \rightarrow \mathrm{i}^{m} u_{n, m}
\end{gathered}
$$

and mirror reflections of the lattice:

$$
\begin{aligned}
m \rightarrow 1-m, \quad \text { i.e. } \quad u_{n, m} \longleftrightarrow u_{n, m+1}, \quad u_{n+1, m} \longleftrightarrow u_{n+1, m+1}, \\
n \rightarrow 1-n, \quad \text { i.e. } \quad u_{n, m} \longleftrightarrow u_{n+1, m}, \quad u_{n, m+1} \longleftrightarrow u_{n+1, m+1} .
\end{aligned}
$$

Now, in the next section we describe the results of this search.

\section{Results}

In this section we describe the results of the procedure outlined in Section 2. Specifically, as described in 2.3 we will explicit the particular cases in which the parametric equations can be divided.

\subsection{List 1}

Equation (E.1): To equation (E.1) correspond only degenerate quad-equations in the sense of condition (2.15).

Equation (E.2): To equation (E.2) correspond two nontrivial quad-equations:

$$
u_{n+1, m+1}\left(a u_{n, m+1}+u_{n, m}\right)-u_{n+1, m}\left(u_{n, m+1}+a u_{n, m}\right)=0, \quad a^{2}=-1 .
$$

Up to transformation (T.1) we can reduce these two equations to only one, say the one with $a=-\mathrm{i}$. Equation (3.1, $a=-\mathrm{i})$ is a special case of equation (7) with $a_{2}=-\mathrm{i}$ of List 4 in [14]. This means that this is a LT equation, its first integrals being:

$$
W_{1}=u_{n+1, m} u_{n-1, m}, \quad W_{2}=\mathrm{i}^{n+1} \frac{u_{n, m+1}-u_{n, m}}{u_{n, m+1}+u_{n, m}} .
$$

See [14] for more details.

Equation (E.3): To equation (E.3) correspond only degenerate quad-equations in the sense of condition (2.15). 
Equation (E.4): To equation (E.4) correspond only degenerate quad-equations in the sense of condition (2.15) or the linear discrete wave equation:

$$
u_{n+1, m+1}+u_{n, m+1}-u_{n+1, m}-u_{n, m}=0 .
$$

Equation (E.5): Equation (E.5) depends on the parameters $a$ and $b$. If $a \neq 0$ it is possible to scale it to one through a scaling transformation. This amounts to consider the cases $a=1$ and $a=0$.

To equation (E.5, $a=1$ ) correspond only degenerate quad-equations in the sense of condition (2.15) or the linear discrete wave equation (3.3). The same holds true for equation (E.5, $a=0$ ). So to all the instances of equation (E.5) correspond only trivial or linear equations.

Equation (E.6): To equation (E.6) correspond two quad-equation. One is the trivial exponential wave equation (1.8a), while the other one is the LT equation:

$$
u_{n+1, m+1} u_{n, m+1}+u_{n+1, m} u_{n, m}+1=0 .
$$

Equation (3.4) is equation (9) with $c_{4}=1$ of List 4 in [14]. Its first integrals are [14]:

$$
W_{1}=(-1)^{m}\left(2 u_{n+1, m} u_{n, m}+1\right), \quad W_{2}=\left(\frac{u_{n, m+1}}{u_{n, m-1}}\right)^{(-1)^{n}} .
$$

Equation (E.1'): To equation (E.1 $\left.1^{\prime}\right)$ correspond only degenerate quad-equations in the sense of condition (2.15) and the trivial linearizable equation (1.8a).

Equation $\left(\right.$ E. $\left.2^{\prime}\right)$ : Equation $\left(E .2^{\prime}\right)$ is very rich, since it give raise to many different equations. We have two trivial linearizable equations of the form (1.8a), but also twelve nontrivial equations. We have four equations of the form:

$$
\left(k_{1} u_{n, m}+k_{3} u_{n, m+1}\right) u_{n+1, m}+\left(k_{2} u_{n, m}+k_{4} u_{n, m+1}\right) u_{n+1, m+1}=0,
$$

with

$$
\begin{array}{llll}
k_{1}=1, & k_{2}=-1, & k_{3}=1, & k_{4}=1, \\
k_{1}=1, & k_{2}=\mathrm{i}, & k_{3}=-\mathrm{i}, & k_{4}=-1, \\
k_{1}=-1, & k_{2}=\mathrm{i}, & k_{3}=-\mathrm{i}, & k_{4}=1, \\
k_{1}=-1, & k_{2}=-1, & k_{3}=1, & k_{4}=-1 .
\end{array}
$$

Then four equations of the form:

$$
u_{n, m} u_{n+1, m}+k_{1} u_{n, m+1} u_{n+1, m}+k_{2} u_{n, m+1} u_{n+1, m+1}=0,
$$

with

$$
\begin{array}{ll}
k_{1}=-1+\mathrm{i}, & k_{2}=-\mathrm{i}, \\
k_{1}=1-\mathrm{i}, & k_{2}=-\mathrm{i}, \\
k_{1}=-1-\mathrm{i}, & k_{2}=\mathrm{i}, \\
k_{1}=1+\mathrm{i}, & k_{2}=\mathrm{i} .
\end{array}
$$


Finally we have four equations of the form:

$$
u_{n, m} u_{n+1, m}+k_{1} u_{n, m} u_{n+1, m+1}+k_{2} u_{n, m+1} u_{n+1, m+1}=0,
$$

with:

$$
\begin{array}{ll}
k_{1}=-1+\mathrm{i}, & k_{2}=-\mathrm{i}, \\
k_{1}=1-\mathrm{i}, & k_{2}=-\mathrm{i}, \\
k_{1}=-1-\mathrm{i}, & k_{2}=\mathrm{i}, \\
k_{1}=1+\mathrm{i}, & k_{2}=\mathrm{i} .
\end{array}
$$

Up to the transformations (T.1,T.3) and (T.1) we can reduce equations $(3.6,3.8,3.10)$ to only two equations, say (3.6) with the parameters (3.7d) and (3.8) with the parameters (3.9d):

$$
\begin{gathered}
\left(u_{n, m}-u_{n, m+1}\right) u_{n+1, m}+\left(u_{n, m+1}+u_{n, m}\right) u_{n+1, m+1}=0, \\
{\left[u_{n, m}+(1+\mathrm{i}) u_{n, m+1}\right] u_{n+1, m}+\mathrm{i} u_{n+1, m+1} u_{n, m+1}=0 .}
\end{gathered}
$$

For a complete description of the transformations leading to (3.12) see Figure 1.

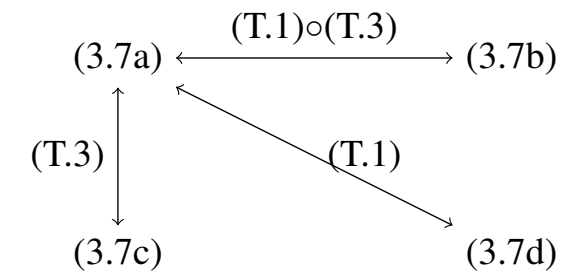

(a) Transformations for equation (3.6)

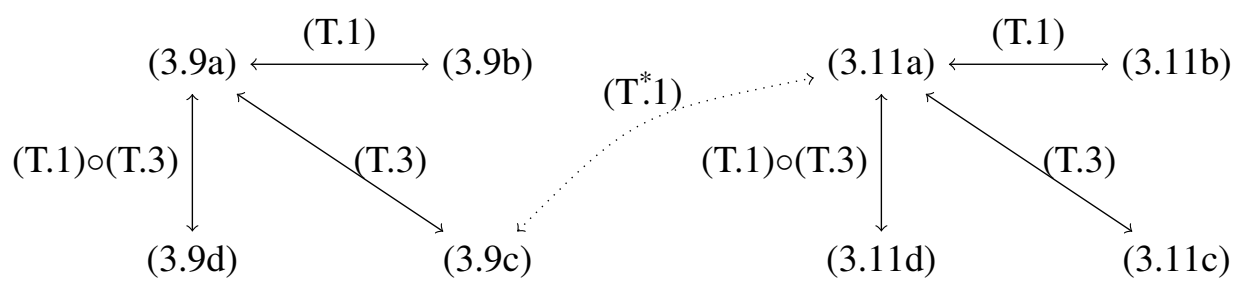

(b) Transformations for equations $(3.8,3.10)$

Figure 1: The relationship between the equations (3.6,3.8,3.10). By (T.1)o(T.3) we mean the composition of the two transformations.

Equation (3.12a) is a LT equation. It can identified with equation (7) with $a_{2}=1$ of List 4 of [14]. In particular its first integrals are given by:

$$
W_{1}=(-1)^{m} u_{n+1, m} u_{n-1, m}, \quad W_{2}=\mathrm{i}^{n} \frac{u_{n, m}+\mathrm{i} u_{n, m+1}}{\mathrm{i} u_{n, m}+u_{n, m+1}} .
$$

Equation (3.12b) is a LT equation too. Its first integrals are given by:

$$
W_{1}=u_{n, m} u_{n+1, m} u_{n+2, m} u_{n+3, m}, \quad W_{2}=(-\mathrm{i})^{n} \frac{u_{n, m}+\mathrm{i} u_{n, m+1}}{u_{n, m}+u_{n, m+1}} .
$$


Note that the $W_{2}$ first integral of (3.12b) is very similar to the $W_{2}$ first integral of (3.12a). Moreover, the $W_{1}$ first integral of (3.12b) is a generalization of the $W_{1}$ first integral of (3.12a) with more points. It is possible to show that equation (3.12b) do not belong to known families of Darboux integrable equations $[5,14,24,37,38]$, because of the specific form of $W_{1}$. Therefore we believe that equation (3.12b) is a new Darboux integrable equation.

\subsection{List 2}

Equation (E.7): Equation (E.7) depends on four parameters $a, b, c$ and $d$ linked among themselves by the condition $(a+1) d=b c$. Therefore we have that using a linear transformation $u_{n, m} \rightarrow \alpha u_{n, m}+$ $\beta$ we need to consider only three different cases: 1) $a \neq 0, a \neq-1, b=0, d=0,2) a=-1, b=1$, $c=0,3) a=-1, b=0$ (recall that $a \neq 0$ in all cases).

Case $a \neq 0, a \neq-1, b=0, d=0$ : For these values of the parameters we obtain either linear equations or exponential wave equations (1.8b) if also $a \neq 1$ and $c \neq 0$. For $a=1$ and $c=0$ we obtain the equation:

$$
\begin{aligned}
& d_{3}^{4} u_{n, m} u_{n+1, m}+d_{3}^{3} d_{4}\left(u_{n+1, m}+u_{n, m}\right)-c_{3} d_{3} d_{4}^{2}\left(u_{n, m+1}+u_{n+1, m+1}\right) \\
+ & d_{3}^{2} d_{4}\left[d_{4}+c_{3}\left(u_{n, m} u_{n+1, m+1}+u_{n, m+1} u_{n+1, m}\right)\right]+c_{3}^{2} d_{4}^{2} u_{n, m+1} u_{n+1, m+1}=0 .
\end{aligned}
$$

In equation (3.15) we have the condition $c_{3} d_{3} d_{4} \neq 0$. Using a linear transformation of the form $u_{n, m}=A \widehat{u}_{n, m}+B$ we can reduce equation (3.15) to two different cases.

Case 1: If $d_{3}^{2}+c_{3} d_{4}=0$ and if we chose $A=d_{4} / d_{3}=-4 B$, we have:

$$
\left(u_{n+1, m+1}-u_{n+1, m}\right)\left(u_{n, m}-u_{n, m+1}\right)+u_{n+1, m+1}+u_{n+1, m}+u_{n, m+1}+u_{n, m}=0 .
$$

This equation is equation (1) of List 3 of [14], hence it is a LT equation with the following first integrals:

$$
\begin{aligned}
& W_{1}=\frac{2\left(u_{n+1, m}+u_{n, m}\right)+1}{\left(u_{n+2, m}-u_{n, m}\right)\left(u_{n+1, m}-u_{n-1, m}\right)}, \\
& W_{2}=(-1)^{n} \frac{u_{n, m-1}+u_{n, m+1}-2\left(u_{n, m}-1\right)}{u_{n, m+1}-u_{n, m-1}} .
\end{aligned}
$$

Case 2: If $d_{3}^{2}+c_{3} d_{4} \neq 0$ we chose

$$
A=2 \frac{d_{4}}{d_{3}}, \quad B=\frac{d_{3} d_{4}}{d_{3}^{2}+c_{3} d_{4}},
$$

and equation (3.15) reduces to:

$$
\left(\frac{c_{3} d_{4}}{d_{3}^{2}} u_{n+1, m+1}+u_{n+1, m}\right)\left(\frac{c_{3} d_{4}}{d_{3}^{2}} u_{n, m+1}+u_{n, m}\right)+u_{n+1, m}+u_{n, m}+\frac{d_{3}^{2}}{d_{3}^{2}+c_{3} d_{4}}=0 .
$$

This equation is equation (3) of List 3 of [14] with $a_{3}=c_{3} d_{4} / d_{3}^{2}$, hence it is a LT equation with the following first integrals:

$$
W_{1}=\left(-a_{3}\right)^{-m} \frac{\left(a_{3}+1\right)\left(u_{n+1, m}+u_{n, m}\right)+1}{\left(u_{n+2, m}-u_{n, m}\right)\left(u_{n+1, m}-u_{n-1, m}\right)},
$$


R.N. Garifullin et al. / Integrable quad-equations admitting known five-point generalized symmetries

$$
W_{2}=\left[\frac{u_{n, m-1}+a_{3} u_{n, m}+1}{\sqrt{-a_{3}}\left(u_{n, m}+a_{3} u_{n, m+1}\right)}\right]^{(-1)^{n}} .
$$

Case $a=-1, b=1, c=0$ : For this value of the parameters only degenerate or linear equations arise. In particular we have discrete wave-like equation.

Case $a=-1, b=0$ : For this value of the parameters we have several linear wave-like equations, but also one nontrivial equation:

$$
\left(u_{n+1, m+1}+\frac{d_{3}}{c_{3}}\right)\left(u_{n, m}-\frac{b_{4}}{c_{3}}\right)-\left(u_{n, m+1}+\frac{d_{3}}{c_{3}}\right)\left(u_{n+1, m}-\frac{b_{4}}{c_{3}}\right)=0,
$$

where we have the following relationship between the parameters:

$$
d=-c \frac{b_{4}}{c_{3}}, \quad c\left(b_{4}+d_{3}\right)=0 .
$$

The case $b_{4}=-d_{3}$ (3.21) is equivalent to (1.8) up to a linear transformation. The case $b_{4} \neq$ $-d_{3}, c=d=0$, and (3.21) is equavalent to (4) of List 4 of [14] up to a linear transformation and it is a LT equation.

Equation (E.8): To equation (E.8) correspond only degenerate quad-equations in the sense of condition (2.15) and the trivial linearizable equation (1.8b).

\subsection{List 3}

Equation (E.3'): To equation (E.3') correspond only degenerate quad-equations in the sense of condition (2.15) and the trivial linearizable equation (1.8a).

Equation (E. $\left.4^{\prime}\right)$ : Equation (E. $\left.4^{\prime}\right)$ depends on the parameters $a$ and $c$. If $a \neq 0$ then it can always be rescaled to 1, hence we can consider two independent cases: $a=1$ and $a=0$.

However, to equation (E. $4^{\prime}$ ) with $a=0$ correspond only degenerate quad-equations in the sense of condition (2.15) and the trivial linearizable equation (1.8a).

On the other hand, the case when $a=1$ admits nontrivial solutions. Indeed, to equation (E. $4^{\prime}$ ) with $a=1$ correspond eight nontrivial equations. Four of them are valid for every value of $c$ :

$$
\left(u_{n+1, m}+\sigma_{1}\right)\left(u_{n, m}-\sigma_{1}\right)=\left(u_{n+1, m+1}-\sigma_{2}\right)\left(u_{n, m+1}+\sigma_{2}\right), \quad \sigma_{i}= \pm 1 .
$$

Using the transformation $u_{n, m} \rightarrow-u_{n, m}$ we can reduce the $\left(3.23,-\sigma_{1}=\sigma_{2}=1\right)$ case to the $\left(3.23, \sigma_{1}=-\sigma_{2}=1\right)$ case and the $\left(3.23, \sigma_{1}=\sigma_{2}=-1\right)$ case to the $\left(3.23, \sigma_{1}=\sigma_{2}=1\right)$ case respectively. This means that we have only the two following independent equations

$$
\begin{aligned}
& \left(u_{n+1, m}+1\right)\left(u_{n, m}-1\right)=\left(u_{n+1, m+1}-1\right)\left(u_{n, m+1}+1\right), \\
& \left(u_{n+1, m}+1\right)\left(u_{n, m}-1\right)=\left(u_{n+1, m+1}+1\right)\left(u_{n, m+1}-1\right) .
\end{aligned}
$$


Other four equations are found if $c=-4$ :

$$
\left(u_{n+1, m}+\sigma_{1}\right)\left(u_{n, m}-\sigma_{1}\right)=-\left(u_{n+1, m+1}-\sigma_{2}\right)\left(u_{n, m+1}+\sigma_{2}\right), \quad \sigma_{i}= \pm 1 .
$$

Using the transformation $u_{n, m} \rightarrow-u_{n, m}$ we can reduce the $\left(3.25,-\sigma_{1}=\sigma_{2}=1\right)$ case to the $\left(3.25, \sigma_{1}=-\sigma_{2}=1\right)$ case and the $\left(3.25, \sigma_{1}=\sigma_{2}=-1\right)$ case to the $\left(3.25, \sigma_{1}=\sigma_{2}=1\right)$ case respectively. This means that we have only the two following independent equations

$$
\begin{aligned}
& \left(u_{n+1, m}+1\right)\left(u_{n, m}-1\right)=-\left(u_{n+1, m+1}+1\right)\left(u_{n, m+1}-1\right), \\
& \left(u_{n+1, m}+1\right)\left(u_{n, m}-1\right)=-\left(u_{n+1, m+1}-1\right)\left(u_{n, m+1}+1\right) .
\end{aligned}
$$

Equation (3.24a) is a well-known sGT equation. It corresponds to equation $T 1^{*}$ in [32]. See references therein for a discussion of the appearance of this equation in literature. On the other hand equation (3.24b) is transformed into equation (3) of List 4 in [14] with the linear transformation $u_{n, m}=2 \widehat{u}_{n, m}+1$. This means that this equation is a known LT equation with the following first integrals:

$$
W_{1}=\left(u_{n+1, m}+1\right)\left(u_{n, m}-1\right), \quad W_{2}=\frac{\left(u_{n, m+2}-u_{n, m}\right)\left(u_{n, m+1}-u_{n, m-1}\right)}{\left(u_{n, m+2}-u_{n, m+1}\right)\left(u_{n, m}-u_{n, m-1}\right)}
$$

For further details see [37].

Equation (3.26a) is a new example of LT equation and has first integrals:

$$
\begin{aligned}
& W_{1}=(-1)^{m}\left(u_{n+1, m}+1\right)\left(u_{n, m}-1\right), \\
& W_{2}=\frac{\left(u_{n, m+3}-u_{n, m-1}\right)\left(u_{n, m+1}-u_{n, m-3}\right)}{\left(u_{n, m+3}-u_{n, m+1}\right)\left(u_{n, m-1}-u_{n, m-3}\right)} .
\end{aligned}
$$

The integral $W_{2}$ is a sixth order (seven-point) first integral. It can be proved that there is no first integral of lower order in this direction. Examples of equations with first integrals of such a high order, except for a special series of Darboux integrable equations presented in [13], are, at the best of our knowledge, new in the literature.

Now we are going to use a transformation theory presented in [37,47]. We can rewrite (3.26a) in a special form and introduce a new function $v_{n, m}$ as follows:

$$
\frac{u_{n+1, m}+1}{u_{n+1, m+1}+1}=-\frac{u_{n, m+1}-1}{u_{n, m}-1}=v_{n+1, m}
$$

So we have

$$
\begin{gathered}
v_{n, m}=\frac{u_{n, m}+1}{u_{n, m+1}+1}, \quad v_{n+1, m}=-\frac{u_{n, m+1}-1}{u_{n, m}-1}, \\
u_{n, m}=1+2 \frac{v_{n, m}-1}{v_{n+1, m} v_{n, m}+1}, \quad u_{n, m+1}=-1+2 \frac{v_{n+1, m}+1}{v_{n+1, m} v_{n, m}+1} .
\end{gathered}
$$


This is a non-point transformation which is invertible on the solutions of (3.26a). Formula (3.30) allows to rewrite (3.26a) as

$$
\left(v_{n+1, m+1}+1\right)\left(v_{n, m}-1\right)=\left(v_{n+1, m}^{-1}+1\right)\left(v_{n, m+1}^{-1}-1\right),
$$

and its first integals as:

$$
\begin{aligned}
& W_{1}=(-1)^{m} \frac{\left(v_{n+1, m}+1\right) v_{n, m}\left(v_{n-1, m}-1\right)}{\left(v_{n+1, m} v_{n, m}+1\right)\left(v_{n, m} v_{n-1, m}+1\right)}, \\
& W_{2}=\frac{\left(v_{n, m+3} v_{n, m+2} v_{n, m+1} v_{n, m}-1\right)\left(v_{n, m+1} v_{n, m} v_{n, m-1} v_{n, m-2}-1\right)}{\left(v_{n, m+3} v_{n, m+2}-1\right) v_{n, m+1} v_{n, m}\left(v_{n, m-1} v_{n, m-2}-1\right)} .
\end{aligned}
$$

Both first integrals (3.32) are of lowest possible order in their directions. Example (3.31) is still exeptional, even though the order of $W_{2}$ has been lowered to five.

Equation (3.26b) is a sGT equation, as we can prove that it possesses the following second order autonomous generalized symmetries in both directions:

$$
\begin{aligned}
\frac{\mathrm{d} u_{n, m}}{\mathrm{~d} t_{1}}= & \left(u_{n, m}^{2}-1\right)\left[\left(u_{n+1, m}^{2}-1\right)\left(u_{n+2, m}+u_{n, m}\right)\right. \\
& \left.-\left(u_{n-1, m}^{2}-1\right)\left(u_{n, m}+u_{n-2, m}\right)-4\left(u_{n+1, m}-u_{n-1, m}\right)\right], \\
\frac{\mathrm{d} u_{n, m}}{\mathrm{~d} t_{2}}= & \left(u_{n, m}^{2}-1\right)\left(T_{m}-1\right)\left(\frac{u_{n, m+1}+u_{n, m}}{U_{n, m}}+\frac{u_{n, m-1}+u_{n, m-2}}{U_{n, m-1}}\right),
\end{aligned}
$$

where

$$
U_{n, m}=\left(u_{n, m+1}+u_{n, m}\right)\left(u_{n, m}+u_{n, m-1}\right)-2\left(u_{n, m}^{2}-1\right) .
$$

It can be proved by direct computation using the method of $[20,31,32]$ that equation (3.26b) does not admit any autonomous generalized symmetries of lower order. Equation (3.26b) seems to be a new interesting example and deserves a separate study, see [17].

Here we limit ourselves to observe that equation (3.26b) is an integrable equation according to the algebraic entropy test $[7,41,43]$. Indeed, computing the degree of the iterates of equation (3.26b) by using the library ae2d.py $[21,22]$, we obtain the following sequence:

$$
1,2,4,7,11,16,22,29,37,46,56,67,79 \ldots
$$

The sequence (3.35) is fitted by the following generating function:

$$
g(z)=\frac{z^{2}-z+1}{(1-z)^{3}}
$$

Since the generating function (3.36) has only one pole in $z_{0}=1$ and it lies on the unit circle, equation (3.26b) is integrable according to the algebraic entropy criterion. Moreover, due to the presence of $(z-1)^{3}$ in the denominator of the generating function (3.36) we have that growth of equation (3.26b) is quadratic. According to the classification of discrete equations using algebraic entropy [26] we have that equation (3.26b) is supposed to be genuinely integrable and not linearizable. 
Equation (E.5'): To equation (E.5') with $a \neq 0$ corresponds one quad-equation:

$$
\begin{gathered}
\left(u_{n, m}-u_{n, m+1}+\frac{b_{4}}{c_{3}}\right)\left(u_{n+1, m}-u_{n+1, m+1}+\frac{b_{4}}{c_{3}}\right) \\
+\frac{b_{4}+d_{3}}{c_{3}}\left(u_{n, m+1}-u_{n+1, m}-\frac{b_{4}}{c_{3}}-a\right)=0, \quad b_{4}+d_{3} \neq 0 .
\end{gathered}
$$

When $a=0$, to equation (E. $\left.5^{\prime}\right)$ corresponds two quad-equations. One of them is just (3.37) with $a=0$, and the second one is:

$$
\begin{gathered}
\left(u_{n, m}+u_{n, m+1}-\frac{b_{4}}{c_{3}}\right)\left(u_{n+1, m}+u_{n+1, m+1}-\frac{b_{4}}{c_{3}}\right) \\
+\frac{b_{4}-d_{3}}{c_{3}}\left(u_{n, m+1}+u_{n+1, m}-\frac{b_{4}}{c_{3}}\right)=0, \quad b_{4}-d_{3} \neq 0,
\end{gathered}
$$

but in this case we have $b=c=0$.

For any $a$ equation (3.37) is transformed into (T4) of [32] by the following non-autonomous linear point transformation:

$$
u_{n, m}=\widehat{u}_{n, m} \frac{b_{4}+d_{3}}{c_{3}}-n\left(\frac{b_{4}+d_{3}}{2 c_{3}}+a\right)+m \frac{b_{4}-d_{3}}{2 c_{3}},
$$

where $\widehat{u}_{n, m}$ satisfies (T4):

$$
\left(\widehat{u}_{n, m}-\widehat{u}_{n, m+1}+\frac{1}{2}\right)\left(\widehat{u}_{n+1, m}-\widehat{u}_{n+1, m+1}+\frac{1}{2}\right)+\widehat{u}_{n, m+1}-\widehat{u}_{n+1, m}=0 .
$$

We recall that equation (T4) is a sGT equation, with two nontrivial three-point generalized symmetries [32].

In the same way equation (3.38) is another sGT equation which is transformed by linear transformation of $u_{n, m}$ into the special form:

$$
\left(u_{n, m}+u_{n, m+1}+1\right)\left(u_{n+1, m}+u_{n+1, m+1}+1\right)=2\left(u_{n, m+1}+u_{n+1, m}+1\right) .
$$

This equation is a new sGT quad-equation which possesses five-point autonomous generalized symmetries:

$$
\begin{aligned}
\frac{\mathrm{d} u_{n, m}}{\mathrm{~d} \theta_{1}} & =\left(u_{n+2, m}-u_{n-2, m}\right)\left(u_{n+1, m}-u_{n, m}\right)\left(u_{n, m}-u_{n-1, m}\right), \\
\frac{\mathrm{d} u_{n, m}}{\mathrm{~d} \theta_{2}} & =\frac{\left[\left(u_{n, m+1}+u_{n, m}\right)^{2}-1\right]\left[\left(u_{n, m}+u_{n, m-1}\right)^{2}-1\right]}{u_{n, m+1}+2 u_{n, m}+u_{n, m-1}}\left(T_{m}-\mathrm{Id}\right)\left(\frac{1}{U_{n, m}}\right),
\end{aligned}
$$

where

$$
U_{n, m}=\left(u_{n, m+1}+u_{n, m-1}\right)\left(u_{n, m}+u_{n, m-2}\right)+2\left(u_{n, m+1} u_{n, m-1}+u_{n, m} u_{n, m-2}+1\right) .
$$

We note that equation (3.38) possesses also the following non-autonomous three-point generalized symmetry in the $n$-direction:

$$
\frac{\mathrm{d} u_{n, m}}{\mathrm{~d} \theta_{3}}=(-1)^{m}\left(u_{n+1, m}-u_{n, m}\right)\left(u_{n, m}-u_{n-1, m}\right),
$$


and the non-autonomous point symmetry

$$
\frac{\mathrm{d} u_{n, m}}{\mathrm{~d} \theta_{4}}=(-1)^{m} .
$$

On the other side it is possible to prove by direct computation using the method of $[20,31,32]$ that no three-point generalized symmetry exists in the $m$-direction. Hence the generalized symmetry (3.41b) is the lowest order generalized symmetry of equation (3.38) in the $m$-direction.

We remark that equation (3.38) through the following nontrival non-invertible transformation:

$$
\widehat{u}_{n, m}=u_{n, m+1}+u_{n, m},
$$

is transformed into (3.26b), i.e. we have that $\widehat{u}_{n, m}$ satisfies equation (3.26b). The symmetries $(3.41 \mathrm{a}, 3.41 \mathrm{~b})$ are transformed into symmetries $(3.33 \mathrm{a}, 3.33 \mathrm{~b})$ too, but here $\theta_{1}=4 t_{1}, \theta_{2}=2 t_{2}$. However, in case of (3.41a), we can check this fact only by using discrete equation (3.40). This means that only common solutions of (3.41a) and (3.40) are transformed into solutions of (3.33a).

Finally, we underline that equation (3.41b) is a particular case of known example of integrable five-point differential-difference equation presented in [3]. According to this remark we have that equation (3.33b) is a nontrivial modification of a known example.

Equation (E.6'): To equation (E.5) correspond only degenerate quad-equations in the sense of condition (2.15) and the trivial linearizable equation (1.8a).

Equation (E. $\left.7^{\prime}\right)$ : Equation (E. $\left.7^{\prime}\right)$ depends on the parameter $a$. If $a \neq 0$ then it can always be rescaled to 1, hence we can consider two independent cases: $a=1$ and $a=0$. However, in both cases to equation $\left(\right.$ E. $\left.7^{\prime}\right)$ corresponds only degenerate quad-equations in the sense of condition (2.15).

Equation (E.8'): To equation (E.5) correspond only degenerate quad-equations in the sense of condition (2.15) and the trivial linearizable equation (1.8a).

\subsection{List 4}

Equation (E.9) To equation (E.9) correspond only degenerate quad-equations in the sense of condition (2.15).

Equation (E.10) To equation (E.10) correspond only degenerate quad-equations in the sense of condition (2.15) and equations of the form of the linear discrete wave equations (3.3).

Equation $($ E.13'): To equation (E.13') correspond two nontrivial equations:

$$
\begin{aligned}
& u_{n+1, m+1}\left(u_{n, m}-u_{n, m+1}\right)-u_{n+1, m}\left(u_{n, m}+u_{n, m+1}\right)+2=0, \\
& u_{n+1, m}\left(u_{n, m+1}-u_{n, m}\right)-u_{n+1, m+1}\left(u_{n, m+1}+u_{n, m}\right)+2=0 .
\end{aligned}
$$

Equation (3.46b) can be reduced to equation (3.46a) using the transformation (T.1). Using the scaling $u_{n, m}=\sqrt{2} \widehat{u}_{n, m}$ equation (3.46a) is reduced to:

$$
\widehat{u}_{n+1, m+1}\left(\widehat{u}_{n, m}-\widehat{u}_{n, m+1}\right)-\widehat{u}_{n+1, m}\left(\widehat{u}_{n, m}+\widehat{u}_{n, m+1}\right)+1=0,
$$

which is a known sGT equation. Namely equation (3.47) is equation (39) of [14], see [12] for more details. Indeed, from [14] we know that actually equation (3.46a) has a more complicated symmetry 
structure in the $n$ direction and its general five-point symmetry is the following non-autonomous one:

$$
\frac{\mathrm{d} u_{n, m}}{\mathrm{~d} \varepsilon_{1}}=\left(u_{n+1, m} u_{n, m}-1\right)\left(u_{n, m} u_{n-1, m}-1\right)\left(a_{n} u_{n+2, m}-a_{n-1} u_{n-2, m}\right),
$$

where $a_{n}$ is a two-periodic function, i.e. a solution of the difference equation $a_{n+2}=a_{n}$. We note that $a_{n}=1$ is a solution which corresponds to equation (E.13'). Moreover equation (3.46a) has a non-autonomous three-point symmetry in the $m$ direction:

$$
\frac{\mathrm{d} u_{n, m}}{\mathrm{~d} \varepsilon_{2}}=(-1)^{n} \frac{u_{n, m+1} u_{n, m-1}+u_{n, m}^{2}}{u_{n, m+1}+u_{n, m-1}} .
$$

No autonomous three-point symmetry exists for equation (3.46a).

\subsection{List 5}

Equation (E.11) To equation (E.11) correspond only degenerate quad-equations in the sense of condition (2.15).

Equation (E.12) Equation (E.12) depends on two parameters $a$ and $b$. If $a \neq 0$ it can always be scaled to $a=1$, so for this equations we get two different cases: when $a=1$ and when $a=0$.

Case $a=1$ : For this value of the parameter only degenerate or linear equations arise. In particular we have a discrete wave equation (3.3).

Case $a=0$ : For this value of the parameter only degenerate or linear equations arise. In particular we have a discrete wave equation (3.3).

Equation (E.13) Equation (E.13) depends only on the parameter $a$. If $a \neq 0$ it can be scaled to $a=1$ always. Therefore we have to consider the cases $a=1$ and $a=0$.

Case $a=1$ : For this value of the parameter there are two nontrivial equations:

$$
\begin{aligned}
u_{n+1, m+1}\left(u_{n, m+1}+u_{n, m}+1\right)+u_{n, m}\left(u_{n+1, m}+1\right) & =0 \\
u_{n, m+1}\left(u_{n+1, m+1}+u_{n+1, m}+1\right)+u_{n+1, m}\left(u_{n, m}+1\right) & =0 .
\end{aligned}
$$

Up to transformation (T.1) we have that equation (3.50b) reduces to equation (3.50a). Therefore we have only one independent equation. Equation (3.50a) is a sGT equation and appeared as formula (58) in [33].

Case $a=0$ : For this value of the parameter there are six nontrivial equations:

$$
\begin{gathered}
\left(u_{n, m}+u_{n, m+1}\right) u_{n+1, m+1}+u_{n, m} u_{n+1, m}=0, \\
\left(u_{n, m}+u_{n, m+1}\right) u_{n+1, m}+u_{n, m+1} u_{n+1, m+1}=0, \\
\left(\frac{1+\mathrm{i} \sqrt{3}}{2} u_{n, m}+\frac{1-\mathrm{i} \sqrt{3}}{2} u_{n, m+1}\right) u_{n+1, m+1}-u_{n, m} u_{n+1, m}=0, \\
\left(\frac{1-\mathrm{i} \sqrt{3}}{2} u_{n+1, m}+\frac{1+\mathrm{i} \sqrt{3}}{2} u_{n+1, m+1}\right) u_{n, m+1}-u_{n, m} u_{n+1, m}=0,
\end{gathered}
$$


R.N. Garifullin et al. / Integrable quad-equations admitting known five-point generalized symmetries

$$
\begin{gathered}
\left(\frac{1+\mathrm{i} \sqrt{3}}{2} u_{n+1, m}+\frac{1-\mathrm{i} \sqrt{3}}{2} u_{n+1, m+1}\right) u_{n, m+1}-u_{n, m} u_{n+1, m}=0 \\
\left(\frac{1-\mathrm{i} \sqrt{3}}{2} u_{n, m}+\frac{1+\mathrm{i} \sqrt{3}}{2} u_{n, m+1}\right) u_{n+1, m+1}-u_{n, m} u_{n+1, m}=0 .
\end{gathered}
$$

Up to transformations (T.1), (T.2) and(T.1) we have that all the equations in (3.51) can be reduced to (3.51a). The precise relationship between these equations is illustrated in Figure 2. Equation (3.51a) is a known LT equation and it can be identified with (6) with $a_{2}=1$ of List 4 of [14]. Its first integrals are:

$$
W_{1}=u_{n+1, m} u_{n, m} u_{n-1, m}, \quad W_{2}=d^{-n} \frac{u_{n, m}-d u_{n, m+1}}{d u_{n, m}-u_{n, m+1}}, \quad d \equiv-\frac{1+\mathrm{i} \sqrt{3}}{2} .
$$

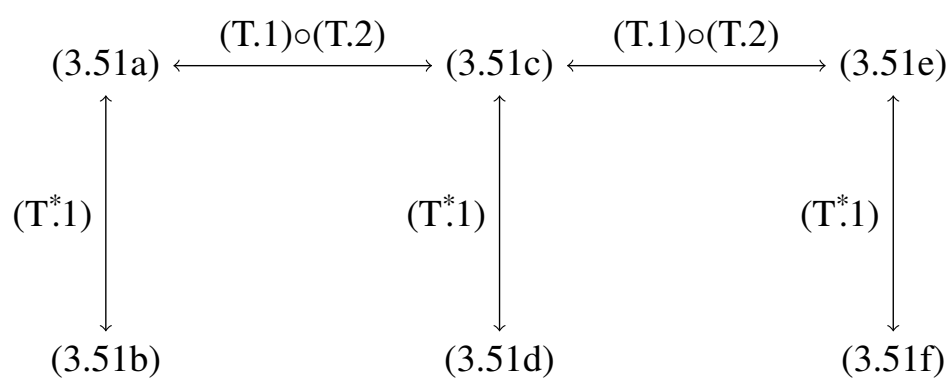

Figure 2: The relationship between the equations (3.51). By (T.1)o(T.2) we mean the composition of the two transformations.

Equation (E.14): To equation (E.14) correspond only degenerate quad-equations in the sense of condition (2.15).

Equation (1.7): To equation (1.7) correspond three nontrivial quad-equations ${ }^{\mathrm{a}}$ :

$$
\begin{gathered}
\left(u_{n+1, m+1}+\frac{c_{4}}{a_{4}}\right)\left(u_{n, m+1}+\frac{c_{4}}{a_{4}}\right)-\left(u_{n+1, m}-\frac{d_{3}}{a_{4}}\right)\left(u_{n, m}-\frac{d_{3}}{a_{4}}\right)=0, \quad a_{4} \neq 0, \\
\left(u_{n+1, m+1}+u_{n+1, m}+\frac{c_{4}}{c_{3}}\right)\left(u_{n, m+1}+u_{n, m}+\frac{c_{4}}{c_{3}}\right)=\frac{c_{4}^{2}-c_{3} d_{4}}{c_{3}^{2}}, \quad c_{3} \neq 0, c_{4}^{2}-c_{3} d_{4} \neq 0, \\
\left(u_{n+1, m+1}-u_{n+1, m}-\frac{c_{4}}{c_{3}}\right)\left(u_{n, m+1}-u_{n, m}-\frac{c_{4}}{c_{3}}\right)=\frac{c_{4}^{2}+c_{3} d_{4}}{c_{3}^{2}}, \quad c_{3} \neq 0, c_{4}^{2}+c_{3} d_{4} \neq 0 .
\end{gathered}
$$

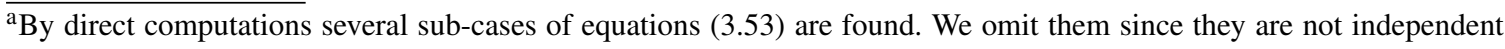
cases. 
Equation (3.53a) is a LT equation. Using the transformation

$$
\widehat{u}_{n, m}=u_{n, m}+\frac{c_{4}}{a_{4}}
$$

we can bring it into equation (1) of List 4 of [14]. Its first integrals are given by:

$$
W_{1}=\left(u_{n+2, m}-u_{n, m}\right)\left(u_{n+1, m}-u_{n-1, m}\right), \quad W_{2}=\left(\frac{a_{4} u_{n, m+1}+c_{4}}{a_{4} u_{n, m}-d_{3}}\right)^{(-1)^{n}} .
$$

Equation (3.53b) under the non-autonomous transformation

$$
\widehat{u}_{n, m}=(-1)^{m}\left(u_{n, m}+\frac{c_{4}}{2 c_{3}}\right)
$$

is mapped into a particular case of equation (3.53c). Equation (3.53c) itself is of LT. It can be identified with equation (5) of List 4 of [14]. This implies that its first integrals are given by:

$$
W_{1}=u_{n+1, m}-u_{n-1, m}, \quad W_{2}=(-1)^{n} \frac{u_{n, m+1}-u_{n, m}+\kappa-\delta}{u_{n, m+1}-u_{n, m}+\kappa+\delta}, \kappa=\frac{c_{4}}{c_{3}}, \delta=\frac{c_{4}^{2}+c_{3} d_{4}}{c_{3}^{2}} .
$$

Equation (E.9'): To equation (E.9') correspond only degenerate quad-equations in the sense of condition (2.15) and the trivial linearizable equation (1.8a).

Equation (E.10'): Equation (E.10') depends on the parameter $a$. If $a \neq 0$ then it can always be rescaled to 1, hence we can consider two independent cases: $a=1$ and $a=0$. However in both cases to equation (E.9') correspond only degenerate quad-equations in the sense of condition (2.15) or linear equations of the form of the discrete wave equation (3.3).

Equation (E.11'): Equation (E.11') depends on the parameter $a$. If $a \neq 0$ then it can always be rescaled to 1, hence we can consider two independent cases: $a=1$ and $a=0$.

To equation (E.11') when $a=1$ correspond four nontrivial equations:

$$
\begin{aligned}
& u_{n, m} u_{n+1, m}+u_{n, m+1} u_{n+1, m}+u_{n, m+1} u_{n+1, m+1}=1, \\
& u_{n, m} u_{n+1, m}-u_{n, m+1} u_{n+1, m}+u_{n, m+1} u_{n+1, m+1}=1, \\
& u_{n, m} u_{n+1, m}+u_{n, m} u_{n+1, m+1}+u_{n, m+1} u_{n+1, m+1}=1, \\
& u_{n, m} u_{n+1, m}-u_{n, m} u_{n+1, m+1}+u_{n, m+1} u_{n+1, m+1}=1 .
\end{aligned}
$$

Up to transformations (T.1,T.1) we have that all the equations in (3.58) can be reduced to (3.58a), see Figure 3. Equation (3.58a) is a known equation. It was introduced in [25], while in [10] it was proved that it is a sGT equation ${ }^{\mathrm{b}}$.

To equation (E.11') when $a=0$ correspond twelve nontrivial equations. Six of them are those of presented in formula (3.51), but we also have the following six other equations:

$$
\begin{gathered}
u_{n, m+1} u_{n+1, m+1}+u_{n+1, m}\left(u_{n, m}-u_{n, m+1}\right)=0 \\
\left(u_{n, m}-u_{n, m+1}\right) u_{n+1, m+1}-u_{n, m} u_{n+1, m}=0
\end{gathered}
$$

\footnotetext{
${ }^{\mathrm{b}}$ Therein it is given by equation (1.9).
} 


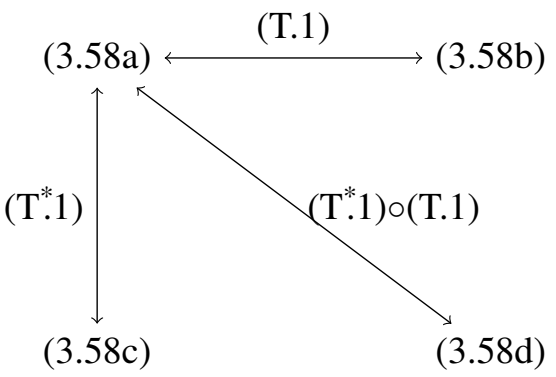

Figure 3: The relationship between the equations (3.58). By (T.1)o(T.1) we mean the composition of the two transformations.

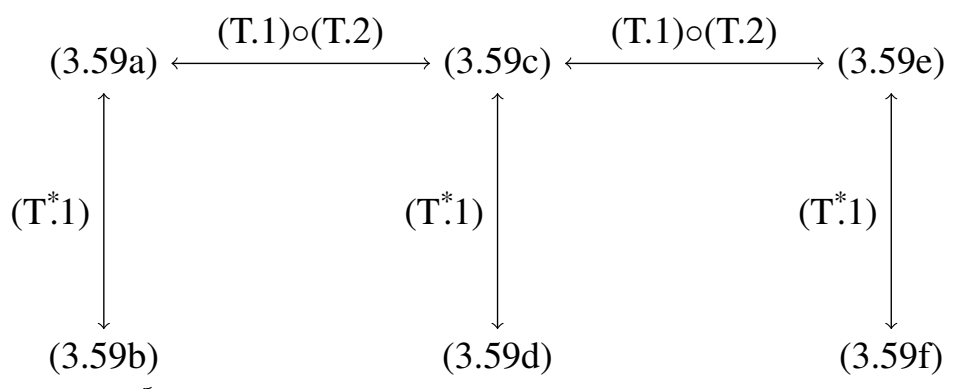

(T.1)

(3.51a)

Figure 4: The relationship between the equations (3.59) and (3.51a). By (T.1)o(T.2) we mean the composition of the two transformations.

$$
\begin{aligned}
& \left(\frac{1-\mathrm{i} \sqrt{3}}{2} u_{n, m+1}+u_{n, m}\right) u_{n+1, m}-\frac{1+\mathrm{i} \sqrt{3}}{2} u_{n, m+1} u_{n+1, m+1}=0 \\
& \left(u_{n, m+1}+\frac{1-\mathrm{i} \sqrt{3}}{2} u_{n, m}\right) u_{n+1, m+1}-\frac{1+\mathrm{i} \sqrt{3}}{2} u_{n+1, m} u_{n, m}=0 \\
& \left(\frac{1+\mathrm{i} \sqrt{3}}{2} u_{n, m+1}+u_{n, m}\right) u_{n+1, m}-\frac{1-\mathrm{i} \sqrt{3}}{2} u_{n, m+1} u_{n+1, m+1}=0 \\
& \left(\frac{1-\mathrm{i} \sqrt{3}}{2} u_{n, m+1}+u_{n, m}\right) u_{n+1, m+1}+\frac{1+\mathrm{i} \sqrt{3}}{2} u_{n+1, m} u_{n, m}=0
\end{aligned}
$$

Using the transformations (T.1,T.1,T.2) we have that the only independent equation is (3.51a). See Figure 4 for the details about the needed transformations. As discussed above equation (3.51a) is a LT equation with known first integrals (3.52). 
Equation (E.12'): To equation (E.12') correspond only degenerate quad-equations in the sense of condition (2.15) or the trivial linearizable equation (1.8a).

\subsection{List 6}

Equation (E.15): To equation (E.15) correspond only degenerate quad-equations in the sense of condition (2.15).

Equation (E.16): To equation (E.16) correspond only degenerate quad-equations in the sense of condition (2.15).

Equation (E.17): Equation (E.17) is not rational, so we are not going to consider it as we are dealing with multi-affine quad-equations (2.3). It can be remarked that equation (E.17) has as a rational form, but it is quadratic in $u_{k \pm 2}$, so it is outside the main class of differential-difference equations.

Equation (E.14'): To equation (E.14') correspond only degenerate quad-equations in the sense of condition (2.15) or equations of the form of the linear discrete wave equation (3.3).

\section{Summary and outlook}

In this paper we have constructed all the possible autonomous quad-equations (1.1) admitting, as generalized symmetries, some known five-point differential-difference equations belonging to a class recently classified in $[18,19]$.

In section 2 we gave a general result on all the quad-equations admitting a generalized symmetry of the form (2.2), resulting in the simplified form (2.3). Then in section 3 we considered all the explicit examples of five-point differential-difference equations and found all the corresponding quad-equations. We discussed the properties of these equations, highlighting which are LT and which are sGT equations. We present a summary of these properties in Table 1. Referring to the table, we have that the majority of the differential-difference equations of the form (1.3) as classified in $[18,19]$ give raise to degenerate or trivial quad-equations. However eight of them give also rise to LT equations. Most of these LT equations were known, but we also obtained two new LT equations, namely equations (3.12b) and (3.26a). Moreover, as expected, sGT equations are even rarer, appearing only in in four examples. Among these sGT equations there are two new examples, namely equations (3.26b) and (3.40). In this paper we limited ourselves to present the result of the algebraic entropy test for equation (3.26b), which suggests integrability. More details and generalizations, including $L-A$ pairs, on equation (3.26b) can be found in [17].

It should be remarked that in $[35,39,40]$ some more general completely discrete equations consistent with the INB-like five-point differential-differential equations were studied. In the mentioned papers a natural discretization based on the Bäcklund transformation was presented and it had the form:

$$
Q\left(u_{n, m+1}, u_{n+1, m+1}, u_{n+2, m+1}, u_{n, m}, u_{n+1, m}, u_{n+2, m}\right)=0 .
$$

This is an equation on two adjacent quads. Quad equations (1.1) like we consider in this paper are also determined by Bäcklund transformations, but of a quite different form. Quad equations (1.1) might be special reductions of equations of the form (4.1) as well of the corresponding Bäcklund transformations. 


\begin{tabular}{|c|c|c|c|}
\hline \multicolumn{2}{|l|}{ Class I } & \multicolumn{2}{|c|}{ Class 2} \\
\hline Equations & Properties & Equations & Properties \\
\hline (E.1) & Trivial & $\left(\mathrm{E} .1^{\prime}\right)$ & Trivial \\
\hline (E.2) & LT & (E.2') & $\mathrm{LT}^{*}$ \\
\hline (E.3) & Trivial & $\left(\mathrm{E} .3^{\prime}\right)$ & Trivial \\
\hline (E.4) & Trivial & $\left(\mathrm{E} .4^{\prime}, a=1\right)$ & $\mathrm{sGT}^{*}, \mathrm{LT}^{*}$ \\
\hline$(\mathrm{E} .5, a=1)$ & Trivial & $\left(\mathrm{E} .4^{\prime}, a=0\right)$ & Trivial \\
\hline$(\mathrm{E} .5, a=0)$ & Trivial & $\left(\mathrm{E} .5^{\prime}\right)$ & $\mathrm{sGT}^{*}$ \\
\hline (E.6) & LT & (E.6') & Trivial \\
\hline$(\mathrm{E} .7, a \neq 0, \pm 1, b=d=0, c \neq 0)$ & Trivial & $\left(\mathrm{E} .7^{\prime}, a=1\right)$ & Trivial \\
\hline$(\mathrm{E} .7, a=1, b=c=d=0)$ & $\mathrm{LT}$ & $\left(\mathrm{E} .7^{\prime}, a=0\right)$ & Trivial \\
\hline$(\mathrm{E} .7, a=-1, b=1, c=0)$ & Trivial & $\left(\right.$ E. $\left.8^{\prime}\right)$ & Trivial \\
\hline$(\mathrm{E} .7, a=-1, b=0)$ & LT & $\left(\right.$ E. $\left.9^{\prime}\right)$ & Trivial \\
\hline (E.8) & Trivial & $\left(\mathrm{E} .10^{\prime}, a=1\right)$ & Trivial \\
\hline (E.9) & Trival & $\left(\mathrm{E} .10^{\prime}, a=0\right)$ & Trivial \\
\hline (E.10) & Trivial & $\left(\mathrm{E} .11^{\prime}, a=1\right)$ & sGT \\
\hline (E.11) & Trivial & $\left(\mathrm{E} .11^{\prime}, a=0\right)$ & LT \\
\hline$(\mathrm{E} .12, a=1)$ & Trivial & $\left(\mathrm{E} .12^{\prime}\right)$ & Trivial \\
\hline$(\mathrm{E} .12, a=0)$ & Trivial & $\left(\mathrm{E} .13^{\prime}\right)$ & sGT \\
\hline$(\mathrm{E} .13, a=1)$ & sGT & (E.14') & Trivial \\
\hline$(\mathrm{E} .13, a=0)$ & $\mathrm{LT}$ & & \\
\hline (E.14) & Trivial & & \\
\hline (E.15) & Trivial & & \\
\hline (E.16) & Trivial & & \\
\hline (E.17) & Not rational & & \\
\hline (1.7) & $\mathrm{LT}$ & & \\
\hline
\end{tabular}

Table 1: Summary of the properties of the corresponding discrete quad-equations. With ${ }^{*}$ we underline the presence of new quad-equations.

As we remarked at the beginning of this section, in this paper we only dealt with autonomous differential-difference equations and autonomous quad-equations. We are now working on weakening this condition for the fully discrete equations to present a classification of non-autonomous quad-equations admitting the differential-difference equations found in $[18,19]$ as five-point generalized symmetries. This classification can be performed with the method presented in [15] and applied to some known autonomous and non-autonomous three-point differential-difference equations of Volterra and Toda type.

\section{Acknowledgment}

GG is supported by the Australian Research Council through Nalini Joshi's Australian Laureate Fellowship grant FL120100094.

We thank the anonymous referee for the interesting comments on the discretization of the INBlike equations. 


\section{Bibliography}

[1] V.E. Adler. Integrable Möbius invariant evolutionary lattices of second order. Preprint on arXiv: 1605.00018.

[2] V.E. Adler. On a discrete analog of the Tzitzeica equation. Preprint on arXiv:1103.5139.

[3] V.E. Adler. Integrable seven-point discrete equations and second-order evolution chains. Theor. Math. Phys., 195, 513-528, 2018.

[4] V.E. Adler and V.V. Postnikov. On vector analogs of the modified volterra lattice. J. Phys. A: Math. Theor., 41, 455203 (16 pp), 2008.

[5] V. E. Adler and S. Ya. Startsev. Discrete analogues of the Liouville equation. Theor. Math. Phys., 121 (2), 1484-1495, 1999.

[6] V.E. Adler, A.I. Bobenko, and Yu.B. Suris. Classification of integrable equations on quad-graphs. The consistency approach. Comm. Math. Phys., 233, 513-543, 2003.

[7] M. Bellon and C.-M. Viallet. Algebraic entropy. Comm. Math. Phys., 204, 425-437, 1999.

[8] O.I. Bogoyavlensky. Integrable discretizations of the kdv equation. Phys. Lett. A, 134, 34-38, 1988.

[9] O.I. Bogoyavlensky. Algebraic constructions of integrable dynamical systems-extensions of the Volterra system. Russ. Math Surveys, 46, 1-64, 1991.

[10] C. Scimiterna, M. Hay, and D. Levi. On the integrability of a new lattice equation found by multiple scale analysis. J. Phys. A: Math. Theor., 47, 265204, 2014.

[11] R.N. Garifullin, I.T. Habibullin, and R.I. Yamilov. Peculiar symmetry structure of some known discrete nonautonomous equations. J. Phys. A: Math. Theor., 48, 235201 (27 pp), 2015.

[12] R.N. Garifullin, A.V. Mikhailov, and R.I. Yamilov. Discrete equation on a square lattice with a nonstandard structure of generalized symmetries. Theor. Math. Phys., 180 (1), 765-780, 2014.

[13] R.N. Garifullin and R.I. Yamilov. Examples of darboux integrable discrete equations possessing first integrals of an arbitrarily high minimal order. Ufa Math. J., 4, 174-180, 2012.

[14] R.N. Garifullin and R.I. Yamilov. Generalized symmetry classification of discrete equations of a class depending on twelve parameters. J. Phys. A: Math. Theor., 45, 345205 (23 pp), 2012.

[15] R.N. Garifullin and R.I. Yamilov. Integrable discrete nonautonomous quad-equations as Bäcklund autotransformations for known Volterra and Toda type semidiscrete equations. J. Phys.: Conf. Ser., 621, 012005 (18 pp), 2015.

[16] R.N. Garifullin and R.I. Yamilov. On integrability of a discrete analogue of Kaup-Kupershmidt equation. Ufa Math. J., 9, 158-164, 2017.

[17] R.N. Garifullin and R.I. Yamilov. An unusual series of autonomous discrete integrable equations on the square lattice, 2018. Preprint on arXiv:1808.05042 [nlin.SI], accepted for publication in Theor. Math. Phys., 200:1, 2019 doi:10.1134/S0040577919070031.

[18] R.N. Garifullin, R.I. Yamilov, and D. Levi. Classification of five-point differential-difference equations. J. Phys. A: Math. Theor, 50, 125201 (27 pp), 2017.

[19] R.N. Garifullin, R.I. Yamilov, and D. Levi. Classification of five-point differential-difference equations II. J. Phys. A: Math. Theor., 51, 065204 (16 pp), 2018.

[20] R.N. Garifullin, E.V. Gudkova, and I.T. Habibullin. Method for searching higher symmetries for quadgraph equations. J. Phys. A: Math. Theor., 44, 325202 (16 pp), 2011.

[21] G. Gubbiotti. Integrability properties of quad equations consistent on the cube. $\mathrm{PhD}$ thesis, Università degli Studi Roma Tre, 2017.

[22] G. Gubbiotti and M. Hay. A SymPy module to calculate algebraic entropy for difference equations and quadrilateral partial difference equations, 2019. in preparation.

[23] G. Gubbiotti, C. Scimiterna, and D. Levi. The non autonomous YdKN equation and generalized symmetries of Boll equations. J. Math. Phys., 58 (5), 053507, 2017.

[24] G. Gubbiotti and R.I. Yamilov. Darboux integrability of trapezoidal $H^{4}$ and $H^{6}$ families of lattice equations I: First integrals. J. Phys. A: Math. Theor., 50, 345205, 2017.

[25] R. Hernández Heredero, D. Levi, and C. Scimiterna. A discrete integrability test based on multiscale analysis, 2013. Preprint on arXiv:1311.1905 [nlin.SI].

[26] J. Hietarinta and C.-M. Viallet. Searching for integrable lattice maps using factorization. J. Phys. A: Math. Theor., 40, 12629-12643, 2007. 
[27] Y. Itoh. An $H$-theorem for a system of competing species. Proc. Japan Acad., 51, 374-379, 1975.

[28] D. Levi. Nonlinear differential-difference equations as Bäcklund transformations. J. Phys. A: Math. Gen., 14, 1083-1098, 1981.

[29] D. Levi, M. Petrera, C. Scimiterna, and R.I. Yamilov. On Miura transformations and Volterra-type equations associated with the Adler-Bobenko-Suris equations. SIGMA, 4, 077 (14 pp), 2008.

[30] D. Levi and R.I. Yamilov. Conditions for the existence of higer symmetries of the evolutionary equations on the lattice. J. Math. Phys., 38 (12), 6648-6674, 1997.

[31] D. Levi and R.I. Yamilov. The generalized symmetry method for discrete equations. J. Phys. A: Math. Theor., 42, 454012 (18 pp), 2009.

[32] D. Levi and R.I. Yamilov. Generalized symmetry integrability test for discrete equations on the square lattice. J. Phys. A: Math. Theor., 44, 145207 (22 pp), 2011.

[33] A.V. Mikhailov and P. Xenitidis. Second order integrability conditions for difference equations: an integrable equation. Lett. Math. Phys., 104, 431-450, 2014, https://doi.org/10.1007/s11005-013-0668-8.

[34] K. Narita. Soliton solution to extended Volterra equation. J. Phys. Soc. Japan, 51, 1682-1685, 1982.

[35] V.G. Papageorgiou and F.W. Nijhoff. On some integrable discrete-time systems associated with the Bogoyavlensky lattices. Phys. A, 228, 172-188, 1996.

[36] O.G. Rasin and P.E. Hydon. Symmetries of integrable difference equations on the quad-graph. Stud. Appl. Math., 119, 253-269, 2007.

[37] S.Ya. Startsev. On non-point invertible transformations of difference and differential-difference equations. SIGMA, 6, 092 (14 pp), 2010.

[38] S.Ya. Startsev. Darboux integrable discrete equations possessing an autonomous first-order integral. $J$. Phys. A: Math. Theor., 47, 105204 (16 pp), 2014.

[39] Yu.B. Suris. Integrable discretizations of the Bogoyavlensky lattices. J. Math. Phys., 37, 3982-3996, 1996.

[40] Yu. B. Suris. The problem of integrable discretization: Hamiltonian approach. Birkhäuser, Basel, 2003.

[41] S. Tremblay, B. Grammaticos, and A. Ramani. Integrable lattice equations and their growth properties. Phys. Lett. A, 278 (6), 319-324, 2001.

[42] S. Tsujimoto and R. Hirota. Pfaffian representation of solutions to the discrete BKP hierarchy in bilinear form. J. Phys. Soc. Jpn., 65, 2797-2806, 1996.

[43] C.-M. Viallet. Algebraic Entropy for lattice equations, 2006. arXiv:0609.043.

[44] P.D. Xenitidis. Integrability and symmetries of difference equations: the Adler-Bobenko-Suris case. In Proceedings of the 4th Workshop Group Analysis of Differential equations and Itegrable Systems, pages 226-242, 2009.

[45] P.D. Xenitidis and V.G. Papageorgiou. Symmetries and integrability of discrete equations defined on a black-white lattice. J. Phys. A: Math. Theor., 42 (35), 454025, 2009.

[46] R.I. Yamilov. Symmetries as integrability criteria for differential difference equations. J. Phys. A., 39, R541-R623, 2006.

[47] R.I. Yamilov. Invertible changes of variables generated by Bäcklund transformations. Theor. Math. Phys., 85, 1269-1275, 1991. 